\title{
Development of Lecture Attendance System for Staff Performance Rating in a Tertiary Institution using Fingerprint Technology
}

\author{
Taiwo Gabriel OMOMULE ${ }^{1}$, Dr. Alaba Olu AKINGBESOTE ${ }^{2}$, Odunayo Olayinka BAYODE ${ }^{3}$ and Gabriel Omojokun AJU $^{4}$ \\ ${ }^{1}$ Assistant Lecturer, Department of Computer Science, Adekunle Ajasin University, Akungba Akoko, Ondo State, NIGERIA \\ ${ }^{2}$ Senior Lecturer, Department of Computer Science, Adekunle Ajasin University, Akungba Akoko, Ondo State, NIGERIA \\ ${ }^{3}$ Graduate, Department of Computer Science, Adekunle Ajasin University, Akungba Akoko, Ondo State, NIGERIA \\ ${ }^{4}$ Lecturer II, Department of Computer Science, Adekunle Ajasin University, Akungba Akoko, Ondo State, NIGERIA
}

${ }^{1}$ Correspondence Author: taiwo.omomule@aaua.edu.ng

\begin{abstract}
Managing time attendance of staff in organizations has proven to be a challenging endeavor. Manual methods have been proposed in capturing employees' attendance but there are inadequacies in measuring the performance of employees. This paper aims at promoting staff efficiency at work by developing a secure attendance management system for use in tertiary institutions via two subsystems; Fingerprint biometrics as a method of identification and authentication and Question-Answering module for staff performance rating. The system uses staff fingerprints stored in the database upon registration for carrying out the process of attendance and validate staff lectures' requirements. It is accentuated by creating a question-answering module that allows students to answer some questions on each available and registered staff, hence, giving a more concentrated content about staff's activities within the school. The evaluation was carried out based on the matching efficiency and attendance accuracy of the proposed system. The proposed system performed excellently with $98.51 \%$ attendance accuracy based on the high successful staff identification recorded. Hence, staff performance rating was generated, thereby creating an avenue for determining promotion grounds.
\end{abstract}

Keyword-- Attendance, Performance, Biometrics, Fingerprint, Question-Answering

\section{INTRODUCTION}

Attendance management of staff in institution can be challenging using the conventional method of paper sheets and old file system method. Every academic institution poses some standards concerning how attendance is to be confirmed for various activities such as student and lecturers in class sessions, laboratory sessions and examination halls, how many times lecturers deliver lectures and other academic duties in the institution [2]. With the rise of globalization, many institutions are perusing different methods and technique that can improve their employees' productivity. The essence is to keep track of attendance of staffs. Many institutions have achieved this through the manual method of paper-based attendance [1].
However, implementing staff attendance in various teaching institutions using this method has become very rigorous and time consuming. Monitoring and computing the average number of lectures delivered for attendance in order to rate their performance based on a time frame has also proven difficult [3].

In big educational institutions, attendance monitoring tends to be faced with great risks which are rampant in verifying staff lecture attendance. Some of the risks include failure to attend classes for maximum number of times, deliver quality lectures with time allotted, forfeiting lecture deadlines, loss of lecture attendance data etc. Moreover, there is low level of efficiency on the use of the attendance data stored for data analytics and usage for promotional processes. Buttressing these issues, academic staffs in some institutions often disregard the importance and competencies to deliver their academic duties mostly in the area of lecture and content delivery. Sometimes, they miss lectures, and other times, the content delivered for students are not up-to-date and therefore lack the basic goal of knowledge-driven education.

The manual method involves lecturers giving an attendance sheet for students to write their names and signatures as a way of confirming their presence for a particular class session. This can help to keep track of the students' attendance, but falsification may occur when a student signs on behalf of his or her colleague as being present in class which can be difficult to prevent especially for large classes where headcount can takes longer time. This also applies to staff attendance monitoring regarding compliance with lecture timetable, number of lectures delivered, teaching readiness, skills, content delivery, relationship with students and other staff, research capability for the essence of performance rating and promotion. A better solution for this manual system of taking staff attendance during lecture delivery, is the use of biometrics characteristics for authentication and verification and question answering module for staff performance rating.

Fingerprint Biometrics is one of the most successful applications of biometric technology. Fingerprint 
identification is the oldest method that has been successfully used in various applications. Each of our ten fingerprints is different from one another and from those of every other persons. Even identical twins have unique fingerprints. That makes them ideal for personal identification[4].

Researchers refer to biometric as a solution for detecting user's identity and security challenges emanating in this modern day. Biometric identification is any automatically measurable, robust and distinctive physical characteristic or personal trait that can be used to identify an individual or verify the claimed identity of an individual [2]. The operational architecture of a typical Biometric System is presented in Figure 1 as follows:

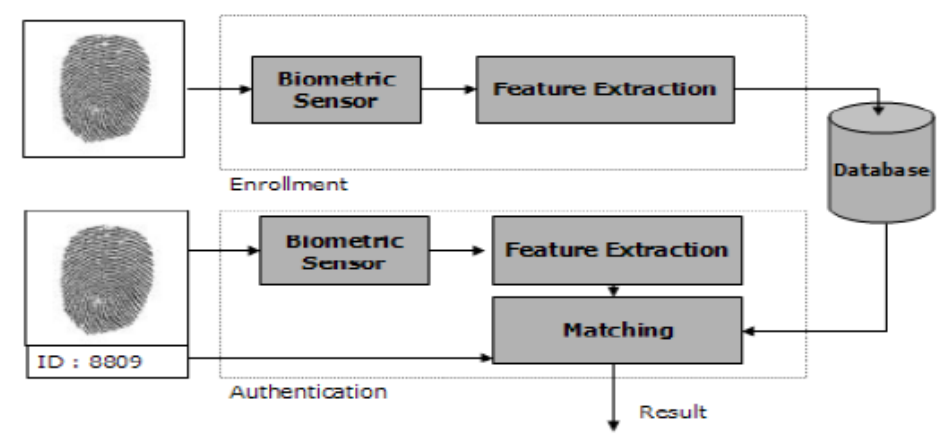

Figure 1: Architecture of Biometric System

Biometric science utilizes the measurements of a person's behavioural characteristics (keyboard strokes, mouse movement) or biological characteristics (fingerprint, iris, nose, eyes, jaw, voice pattern, etc.). A fingerprint is made of a series of ridges and furrows on the surface of the finger. The uniqueness of a fingerprint is determined by the pattern of ridges and furrows as well as the minutiae points. Minutiae points are local ridge characteristics that occur when a ridge splits apart or a ridge ends. Figure 2 presents the various part of a fingerprint [9] as detailed on www.biometrics.gov:

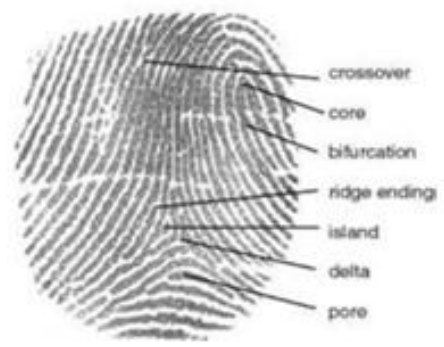

Figure 2: Parts of a Fingerprint

There are several factors that can affect the quality of fingerprints images, these include; manual work, weather condition, contact of finger with sensor, greasy or dirty finger, cuts, wounds or bruises. All these have major or minor impact on the quality of fingerprint images. As such, the fingerprint algorithms procedure delivers the best match between the template fingerprint and query fingerprint for genuine verification and authentication of enrolled individuals. Fingerprint matching module computes a match score between two fingerprints, which should be high for fingerprints from the same finger and low for those from different fingers [9].Fingerprint scanner is an external device generally used for the identification of a person based on unique patterns and ridges of fingerprint as depicted in Figure 3 below:

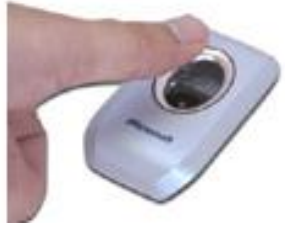

Figure 3: Fingerprint Scanner 
Fingerprint matched a reference number or pin number with a person's name or account [6].It is the features captured that is being transformed digitally into a template. On matching, the computer software compares the new template with the other templates in the database.
When a matching template is found, the staff is identified. This identification and matching process takes under one second to complete. The recognition software can then be used to discover an individual as the person they claim to be [2] as shown in Figure 4 below:

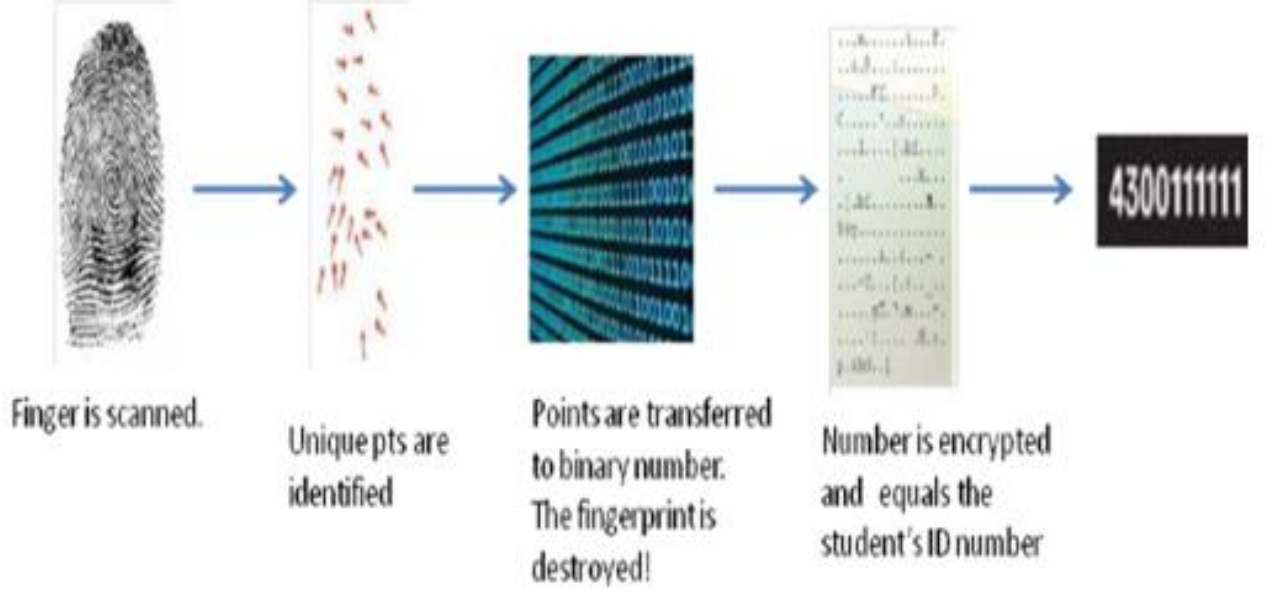

Figure 4: Fingerprint converted digitally into a template

Biometric technology uses automated methods for identifying and verifying a person based on physiological and behavioral traits. Because some parts of the human body are used in biometrics, the issue of getting lost is not possible and for password to be easily guessed can be easily avoided. Also, utilizing biometrics in most cases can be said to be more efficient when speed is considered and convenient than employing password and ID cards method. It is one of the most matured biometric traits and is accepted in courts of law as a legitimate proof of evidence and adopted in forensic analysis globally in the investigations of criminal. More recently, there are growing numbers of individuals and commercial users that are currently using or strongly putting into consideration the use of fingerprint-based identification for no any other reason other than the matching performance biometric technology has demonstrated as well as a better understanding of fingerprints [2].

Therefore, this paper is aimed at developing a staff attendance system to track staff academic activities and the relevance of the content delivered in their lectures over a semester or session that will be used to carry out the performance rating of the staff in the institution.

\section{RELATED WORKS}

Several authors have proposed different techniques and methods in carrying out staff attendance without proper attention to their performance rating. An overview of the fundamentals of biometric identification together with a description of the main biometric technologies currently in use within a common reference framework is presented in
[5]. The research provided an insight into Biometric Identification Systems (BISs) and their potential applications. The general BIS model was proposed for better understanding of biometric identification technologies, and for comparing apparently disparate systems. The work in [5] provided proposal about the use of multi-biometrics such that fusing biometric identification systems (BISs) provides better results than the stand-alone use of each, but it offers no technical detail and implementation of BIS in the domain of application. However, selecting effective multi-biometric techniques that will give the required performance may prove difficult. The authors in [6] proposed an attendance management system for industrial workers using fingerprint scanners so that workers do not get any opportunity to give fake attendance. The objective was to improve the performance of attendance management system based on fingerprint identification for large industrial databases. Three algorithms namely; gender estimation, key-based one-tomany matching and removing boundary minutiae were used in the realization of the system. The system provided accurate attendance information of workers and an interface for workers to communicate with top management authorities but failed to use record of staff attendance to analyze their performance. In [4], wireless fingerprint based college attendance system using Zigbee technology was presented. The objective was to propose a system that takes attendance of student while maintaining their records automatically. The system takes attendance with the help of a fingerprint sensor module and all the records are saved on a computer. Fingerprint sensor module and LCD screen are dynamic which can move in the room. Microsoft SQL 
server database was used to store the records and the user interface was developed with Microsoft C\# programming language using Microsoft Visual Studio framework. The work proposed in [4] offered a robust system for attendance authorization. They are however limited because it was only tested for student lecture attendance. Using other staff attendance factors may limit the performance of the system. Fingerprint Biometric Attendance System for non-academic staff in a tertiary institution was developed in [1]. The system evolved from proposing an efficient system to solve the problem of manual attendance where staff records fake timings in the manual register and yet receiving full payment for the month. The proposed model adopts a process of requirements engineering (RE), to complement Object Oriented Design (OOD) modelling using the Unified Modelling Language (UML) and was implemented using Microsoft Visual Basic.Net. Though the system was able to track and report fake timings recorded by staff, enormous time is often wasted in analyzing the performance of staff in consideration for monthly salary payment. The work in [4] was further extended by the authors in [3] through the inclusion of an SMS module for Wi-Fi supports. They were motivated by the need to simplify student attendance calculation and maintenance using hardware components like fingerprint scanner, Wi-Fi module, Arduino Nano micro-controller and Website for maintaining attendance record. The system showed some measures of reliability for checking student attendance via stored data of fingerprint record in database and for sending an SMS to registered mobile if the person was found absent. However, when tested for staff performance analysis the system may prove unreliable. The authors in [7] proposed a novel fingerprint reconstruction algorithm to reconstruct phase image, which is then converted into the grayscale image. The proposed reconstruction algorithm was used to automate the whole process of taking attendance, manually which is a laborious, troublesome work and time consuming. Results obtained showed that reconstructed images enhance great image error recovery but during matching, there may be rampant failure on mismatch between reconstructed fingerprint matches with the original fingerprint. The authors in [8] proposed a system for student attendance monitoring in a tertiary institution using NFC technology. Their system involved the use of NFC capable student ID cards to mark attendance. Their idea was to ensure that users do not require an NFC enabled phone. The system was implemented using a backend application to generate the identification policies, collect and store data that can be assessed through a web interface. This system just requires a tap to identify a user making it very fast secure to use. This system however as with other NFC based attendance systems does not provide a way to verify the identity of individual students. There are possibilities of students taking attendance on behalf of their friends. Toward creating an efficient biometric employee attendance system, the authors in [9], proposed a system as tested prototype design that will improve upon the existing attendance system in order to foster quality employee productivity. Statistical methods based on oral interview and questionnaire were adopted to collect data about the existing system tending to the design of the proposed system. Although the system recorded success in capturing the employees' attendance but areas with employees' works dedication and promotional criteria were not addressed. The authors in [10] developed staff attendance management system using fingerprint biometric identification technique. The purpose was to present a framework in which attendance management will be automated. The framework performs enrollment and authentication of staff as fast as possible. The implementation was carried out using Microsoft's Visual Studio's C\# Programming Language which is on the .NET platform, with MySQL in the WAMP (Windows, Apache, MySQL, and PHP) Server as the back end. The system was able to curb the problems of tardiness and lateness of lecturers to lecture rooms but no indication as to the rating of staff lecture performance. Wireless attendance management system that authenticates using the iris of the individual was presented in [11]. It was an implementation of off-line iris recognition management system for image capturing, extracting precise details, storing and matching the captured image with one stored in the database. The system was able to address wrong clocking where student inappropriately clocks in for another. The work in presented [11] was found useful in the application domain but users tend to have phobia in the use of Iris scanner because its radioactive properties can cause damage to the eyes. Also in [12], a fingerprint-based attendance management system was presented. The system was designed to also operate as a standalone and handheld system without the use of a computer, unlike other fingerprint attendance systems. The software component used include Visual Basic.Net and MS-Excel to develop the frontend and backend engines while the hardware (device) comprises of the microcontroller, the fingerprint scanner, LCD display, real-time clock and serial communication that is housed separately in a portable box, and used for verification and assigning of time to the registered user and sending the attendance data to the Visual Basic program on the computer. The system also proves reliable but may not stop cases of intentional presentation of another identity. The use of fingerprint technology to achieve effective employee attendance management was presented in [13]. It was carried out in an organization with the sole aim of capturing employees' attendance. The system recorded good results for employees' absenteeism leading to several penalties, but failed to address employees' performance. The authors in [14][15] proposed systems for the authentication, monitoring and control of students' 
attendance. The systems evolved from the integration of fingerprint identification with user-friendly graphical user interface (GUI) to give transparent attendance system, keep real time data and displays online data for parents and other academic use. Though required attendance reports were generated in less time, cases of server downtime often affect authentication error and generation of inaccurate attendance result. Moreover, a high number percentage of parents lacks computing and networking skills to operate the system, thereby required comprehensive training. Also, in [16], a biometric-based Staff Attendance Monitoring System is presented. The aim was to make sure that the staff members are punctual and do their jobs on time. The system was developed using Visual Basic Programming Language as front end while Microsoft Access was used as the Database to the backend users. The system effectively recorded staff attendance as proposed but failed to generate technical performance report for staff utilizing the system. A fingerprint-based authentication system for efficient students' time and attendance management was presented in [17][18]. [17]uses fingerprint technology to authenticate every student based on four main modules namely; fingerprint capture, fingerprint processing, fingerprint matching and database and four deployment structure which consists Fingerprint Terminals, Database Server,
Access Workstation and Network Service. But the authors in [18] relied on a simulation test using biometric sensor hardware to check the fingerprint and compare with the preloaded fingerprint in the database. The simulation test is carried out using MATLAB. The systems proved to be a veritable tool in achieving the much needed automation for attendance management. But breakdown in network service may result to error in authentication, inaccurate attendance report and unverified detection technique.

\section{METHODOLOGY}

The lecturer fingerprint is captured via a fingerprint scanner and the minutiae is extracted and processed. The feature sets are stored in the database as template. On taking another lecture, the lecturer's fingerprint is captured again for verification and also the lecture count is recorded respectively. When there exist a period for promotion or honors for a staff, the performance rating of the staff will be carried out via an online questionanswering survey containing various performance indices and filled by the students. The result will be analyzed and evaluated to validate the staff performance. The architecture of the system is shown in Figure 5 as follows:

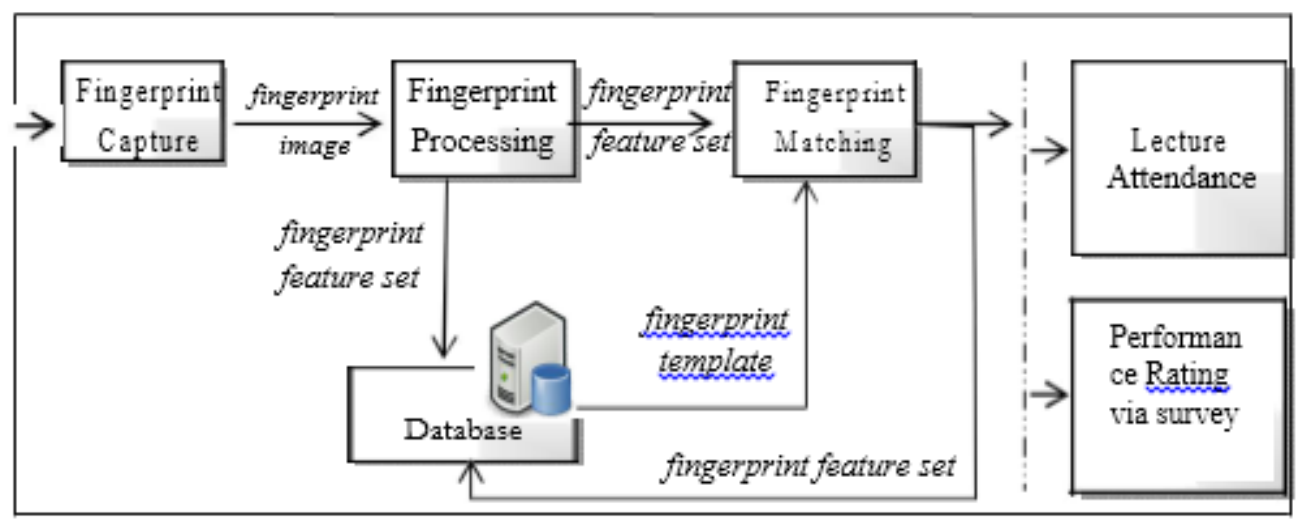

Figure 5: Architecture of the Proposed System (Adapted from Ikuomola, 2015)

The modules in the architecture are presented as follows: Fingerprint Capture: This module interfaces with the fingerprint scanner to capture the fingerprint of the individual to be enrolled or authenticated. This is also termed as the Enrolment Phase. Fingerprint Processing: This module accepts the fingerprint image taken by the sensor and extracts the unique features of the fingerprint (minutiae points) to be used for matching with features saved for the templates in the database. Fingerprint Matching: This module compares the features extracted from the taken (new) fingerprint sample with features of fingerprint templates stored in the database. This is done by performing comparison on a one-to-one basis. Database: The database stores staff fingerprint templates as well as fingerprint history. It also provides data storage for daily lecture attendance records. Lecture Attendance: This module shows the attendance result for each staff while Performance Rating module shows the result of student justification of the performance of staff toward lecture delivery.

\subsection{Mathematical Model}

Given that a total of $N$ staff biometric data are stored in the database and a set of minutiae, $X$ is extracted from a fingerprint at the feature extraction module and 
passed onto the matching module, the Likelihood Ratio

(LLR) is computed as follows:

$$
\begin{gathered}
P(X \mid I=0) \\
L L R=\frac{P(X \mid I=1)}{P(X)}
\end{gathered}
$$

where: $X=\left\{S_{1}, Q_{1}\right\}, \quad S_{1}=$ matching score from fingerprint biometric matching module, $Q_{1}=$ quality of fingerprint biometric sample, $I \in 0,1$ while: $P(X \mid I=0)=$ the genuine distribution of set $X$
$P(X \mid I=1)=$ the imposter distribution of set $X$

In comparing fingerprint biometric data with template data, minutiae matching is computed as follows:

$$
\left(\begin{array}{c}
r_{k}^{T} \\
\phi_{k}^{T} \\
\theta_{k}^{T}
\end{array}\right)\left(\begin{array}{c}
\sqrt{\left(\operatorname{row}_{k}^{T}-\operatorname{row}_{r e f}^{T}\right)^{2}+\left(\operatorname{col}_{k}^{T}-\operatorname{col}_{\text {ref }}^{T}\right)^{2}} \\
\tan ^{-1}\left(\frac{\operatorname{row}_{k}^{T}-\operatorname{row}_{r e f}^{T}}{\operatorname{col}_{k}^{T}-\operatorname{col}_{r e f}^{T}}\right) \\
\theta_{k}^{T}-\theta_{r e f}^{T}
\end{array}\right)
$$

where for a template image: $r_{k}^{T}$ is the radial distance of $k^{\text {th }}$ minutiae, $\emptyset_{k}^{T}$ computes the radial angle of $k^{\text {th }}$ minutiae $\theta_{k}^{T}$ computes the orientation angle of $k^{\text {th }}$ minutiae while $\operatorname{row}_{r e f}^{T}, \operatorname{col}_{r e f}^{T}$ denotes the row, column index of reference points being considered.

\subsection{Modular Flow of the Proposed System}

The system consists of fingerprint acquisition, a fingerprint authentication module and Online Survey. The fingerprint acquisition module is used for capturing the fingerprint from a user through a fingerprint reader. The fingerprint authentication module performs the comparison of a fingerprint template stored in the database with a new fingerprint image acquired from the reader. The online survey gives rooms for students to tell more about staffs in the department. The modular flow of the system is presented in Figure 6 while the overall flowchart of the proposed system is presented in Figure 7 respectively.

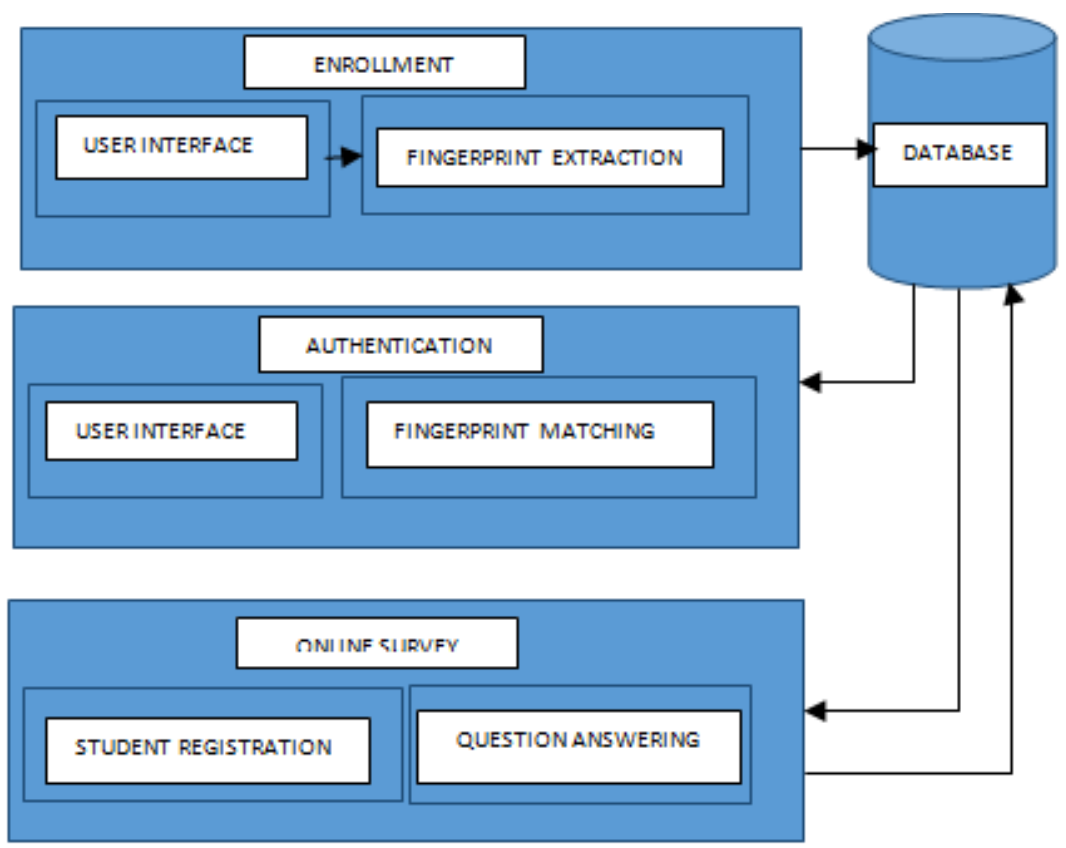

Figure 6: Modular Flow of the System 

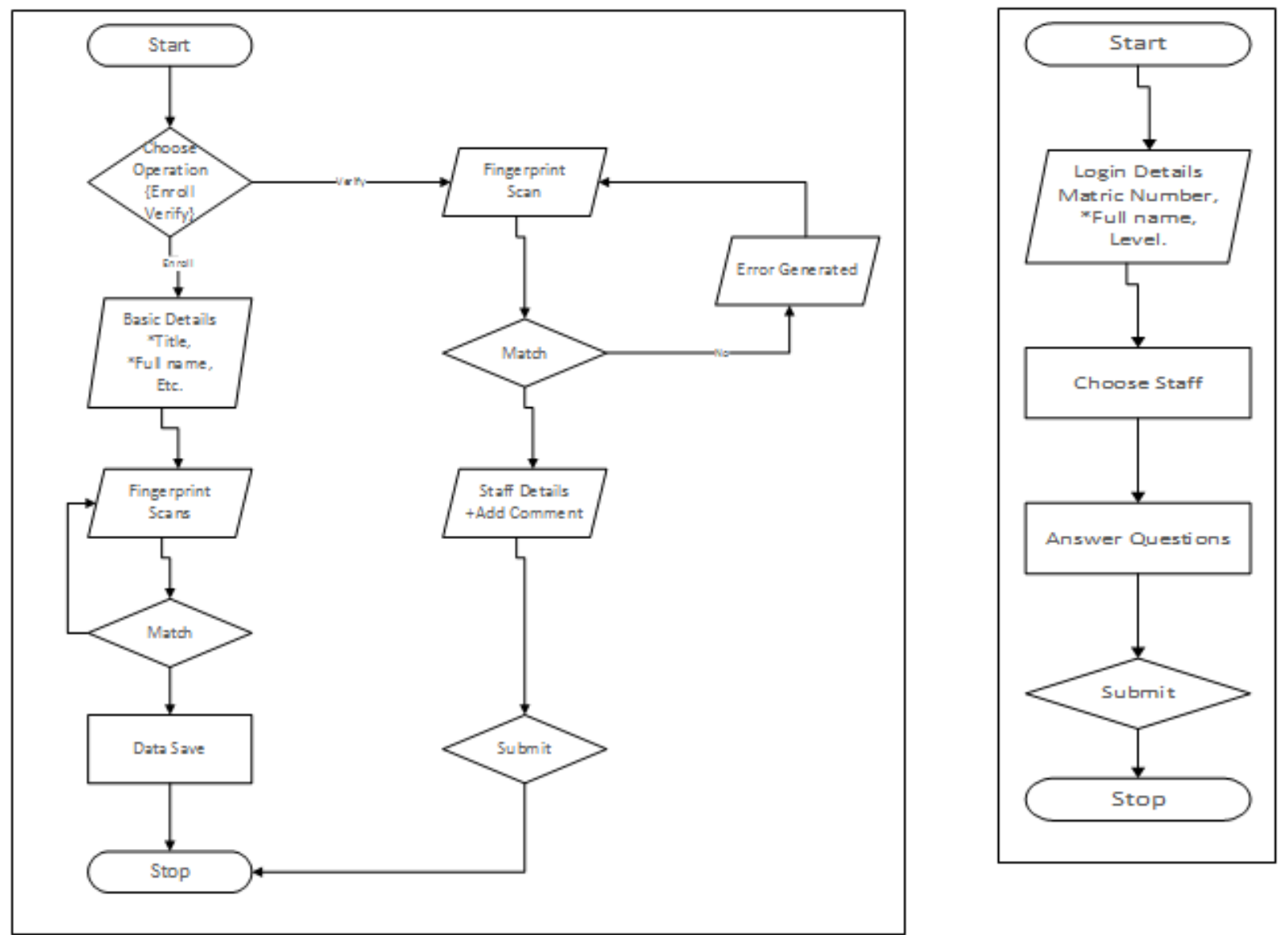

Figure 7a: Flowchart of the Fingerprint Module

Figure 7b: Flowchart of the Question-Answering Module

Figure 7: Overall Flowchart of the Proposed System

\section{IMPLEMENTATION}

The proposed system being a two-way system (Enrollment/Registration and Verification) adopted two approaches to development. The enrollment and verification phase of the system is a desktop-oriented application making use of the Visual C\# framework of the Microsoft .Net. Whereas, the online survey system boasts an implementation for staff performance rating using HTML,CSS, PHP (hypertext preprocessor) and JavaScript framework "React JS" as the initiator for the frontend engines. During registration/enrollment, the staff's brief personal and biometric data are collected from the system and stored in a database. The research work would incorporate the simple, readily available MySQLi database as the backend engine. The software platforms on which the developed system would operate include Wamp Server (MySQLi) version 2.4 or later, Visual Studio IDE, Source AFIS Plugin, GrFinger (Griaule Fingerprint SDK), DigitaPersona Software Development Kit, Operating System (Windows) and Browsers (Chrome or Firefox) while the hardware requirements needed include a computer system with a minimum memory of $256 \mathrm{MB}$ in size, DigitaPersona fingerprint scanner, Hard Disk Size $40 \mathrm{~Gb}$ (Minimum) and other hardware resources. The system testing spurn different stages, and all the stages are presented as follows:

\subsection{Start Up Screen}

This is the first page giving the staffs the opportunity to choose between Enrollment and Verification. The staffs can navigate to their intended part of the system without breaking a sweat. This is shown in Figure 8 below: 


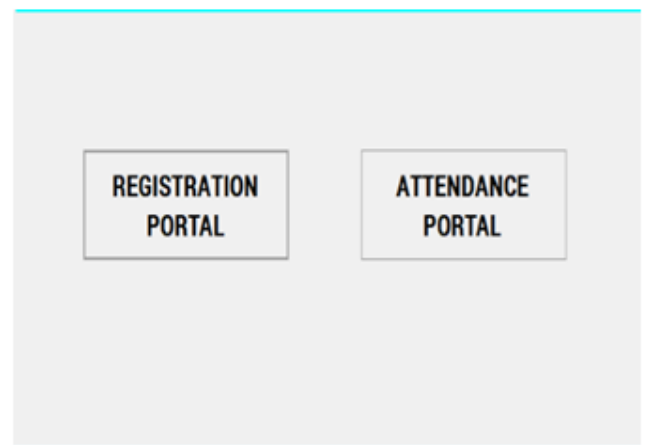

Figure 8: Start-up screen

\subsection{Staff Registration Portal}

Finger 9 indicates an interface where user fingerprint as well as the other bio-data are stored for the first time into the database for staff registration. All data and information required for the proper recording of attendance is enrolled. Personal details and the photo of the staff is first inputted into the system which then prompts the fingerprint acquisition screen to bump out, hence, the fingerprint details of the staff are captured and also stored as shown in Figure 10.

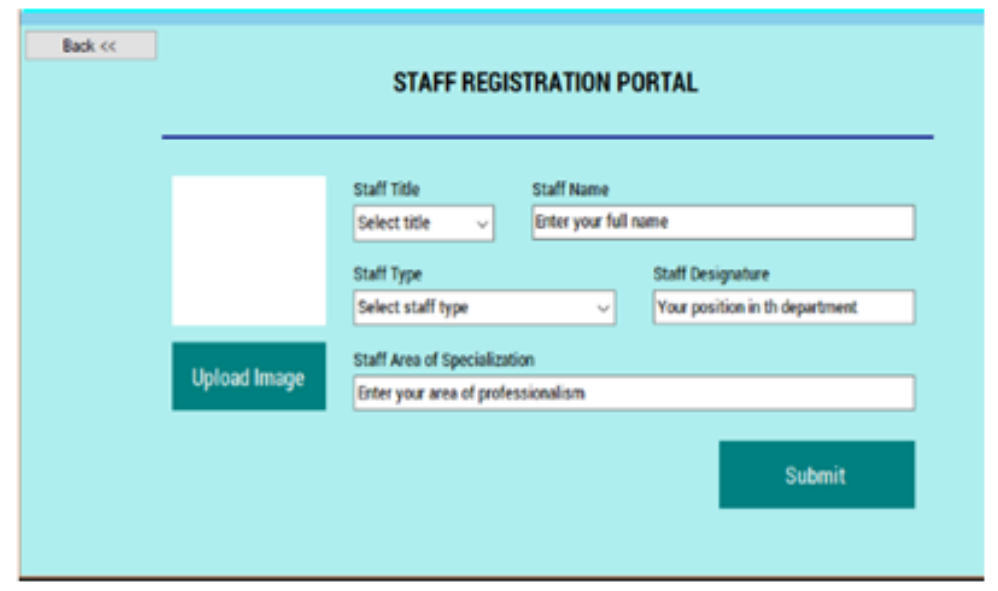

Figure 9: Staff Registration Portal

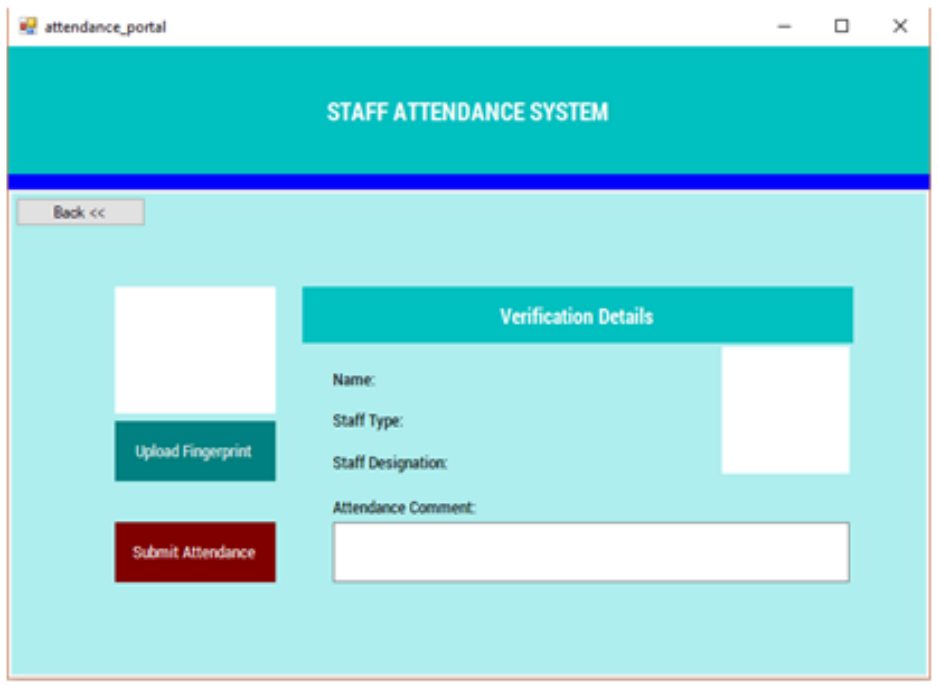

Figure 10: Fingerprint Capture Registration Page 


\subsection{Attendance Verification Page}

After the details of the staff has been obtained from the registration page, the staff registered can then take attendance by placing his/her finger on the fingerprint scanner. The fingerprint image gotten from the scanner is then compared with each fingerprint template on the database. If a match is found, the staff information is retrieved and then displayed on the platform. Before submitting the attendance, the staff can make comment on activity performed that day as a content to his/her attendance. However, if a fingerprint match cannot be found in the database, a prompt of match not found is triggered which can be that there is not fingerprint that correlate with the inputted one. This is presented in Figures 11 and 12 below:

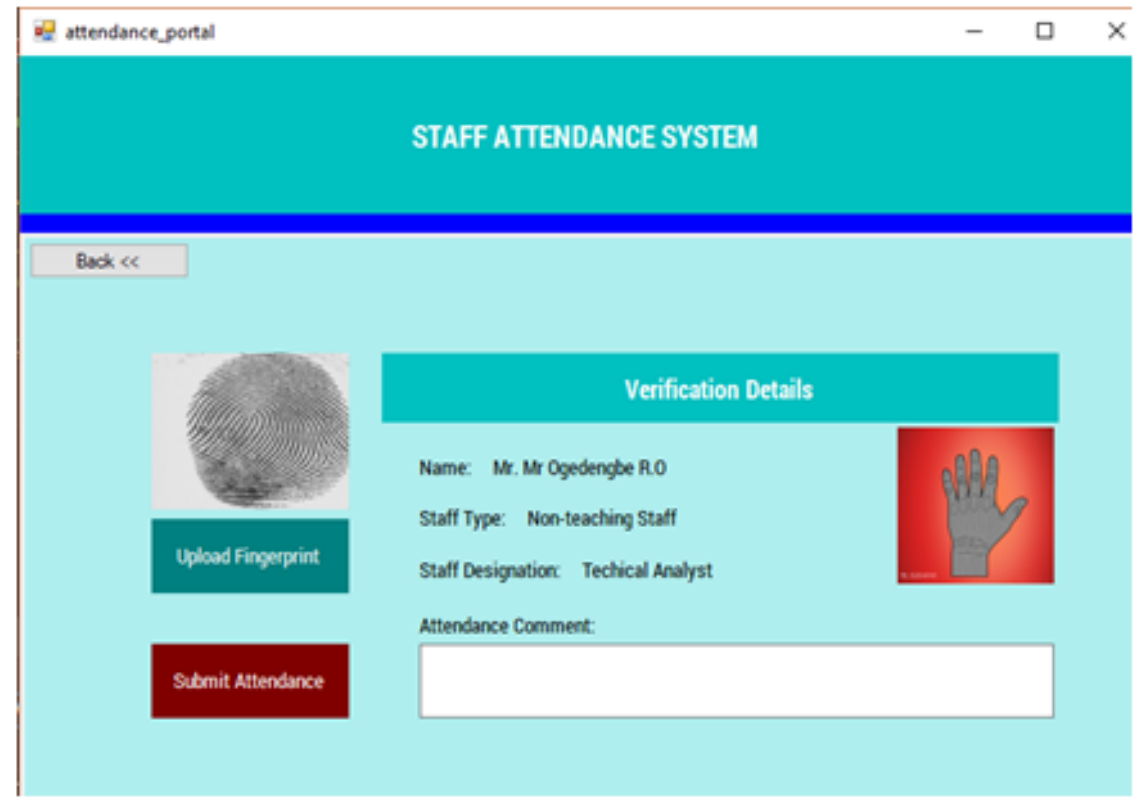

Figure 11: Registered User Verification Page

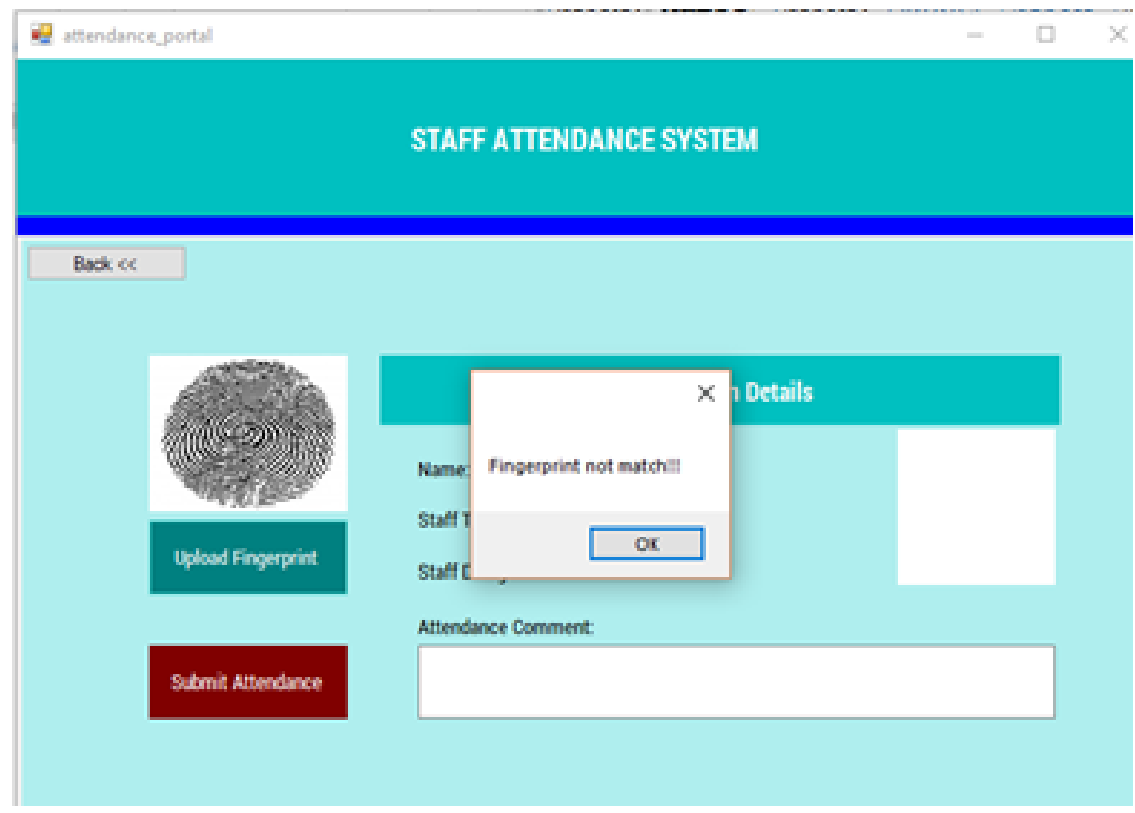

Figure 12: Non-Registered User Verification Page 


\subsection{Question Answering Start Up Screen}

At the end of the month, the students are presented with an online survey system which allows the student to provide adequate information about the staff and general performance and. The Start Up screen is first presented, which shows a login screen for the students to insert their details and then process to feeling the survey question as shown in Figure 13. Figure 14 indicates the login validation form.

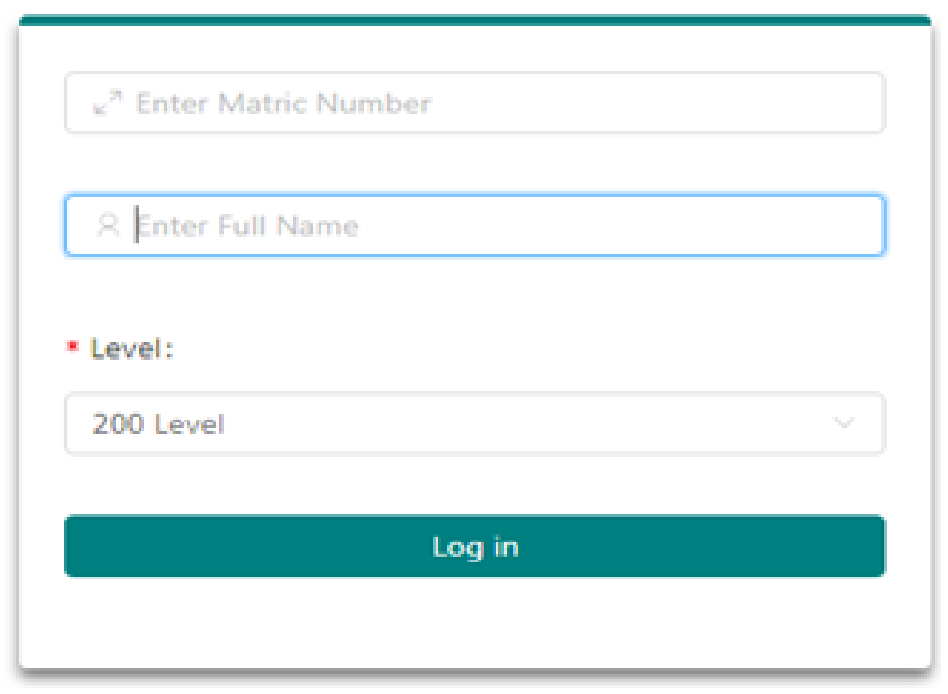

Figure 13: Online Question-Answering

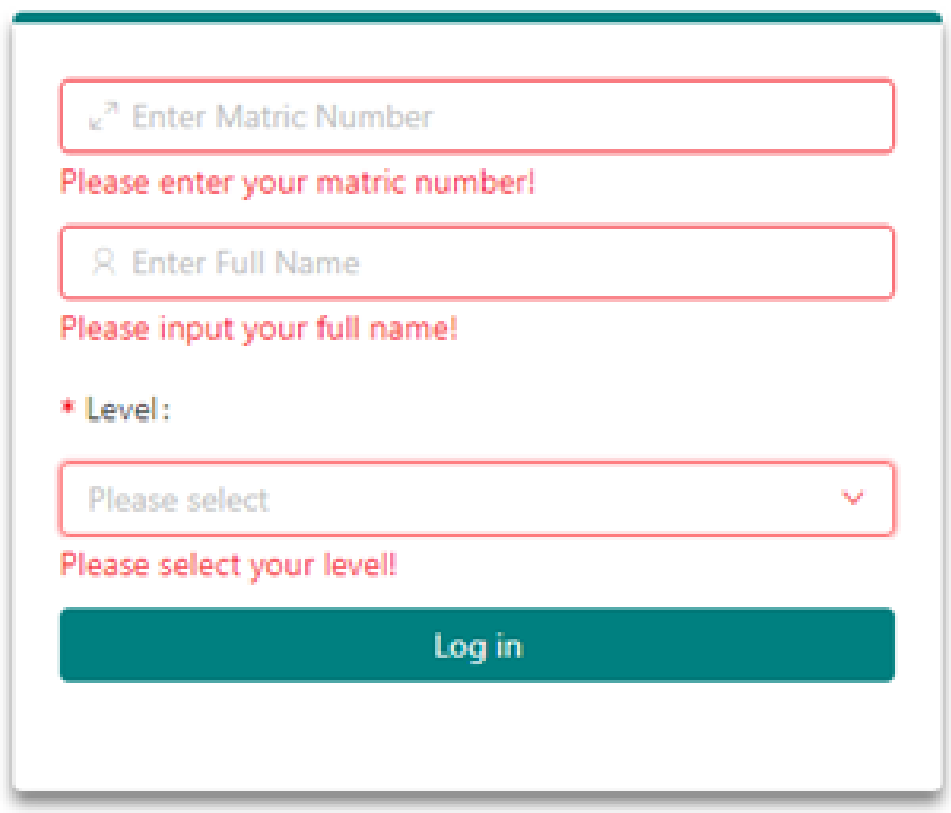

Figure 14: The login Validation

4.5 Question Answering Subsystem (Dashboard)

After a successfully login, the student is presented with a list of staffs from which they could click on. On click on each of the staff brings out a modal of survey question for the students to provide answer to. This is presented in Figure 15. 


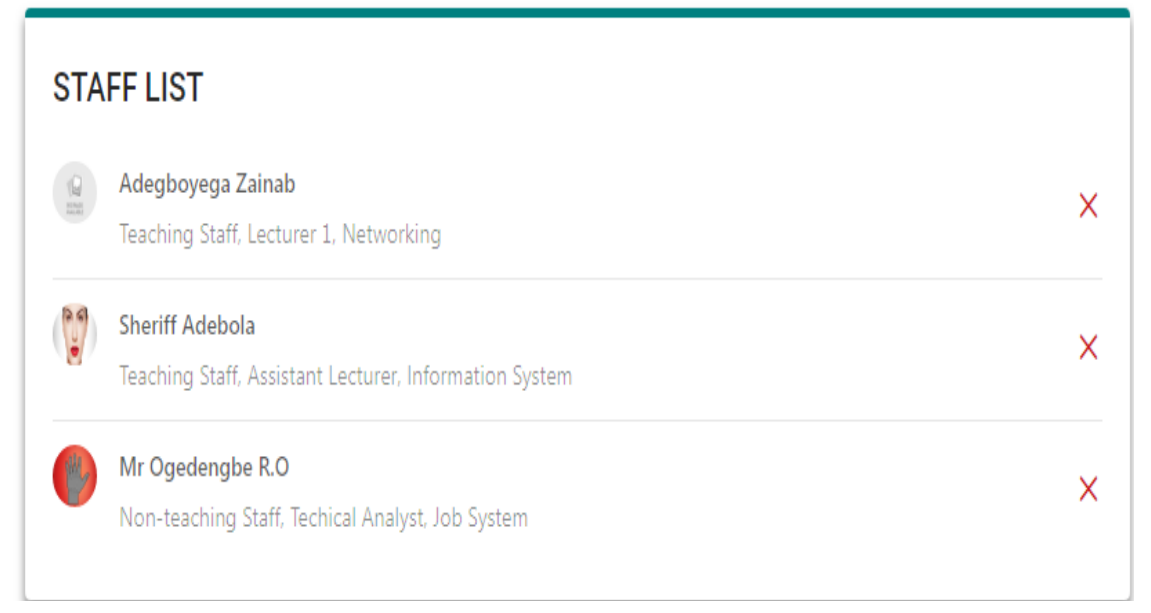

Figure 15: Questionnaire Answering- dashboard showing staff list

\subsection{Question Answering Subsystem (Question Modal)}

In this menu, a list of questions is presented to the students. The questions provided allows the students to tell more about the activities of each individual staffs that had already taken his or are attendance with the system. On successful answering of the provided questions, the student can then submit his/her survey for storage as presented in Figure 16 below:

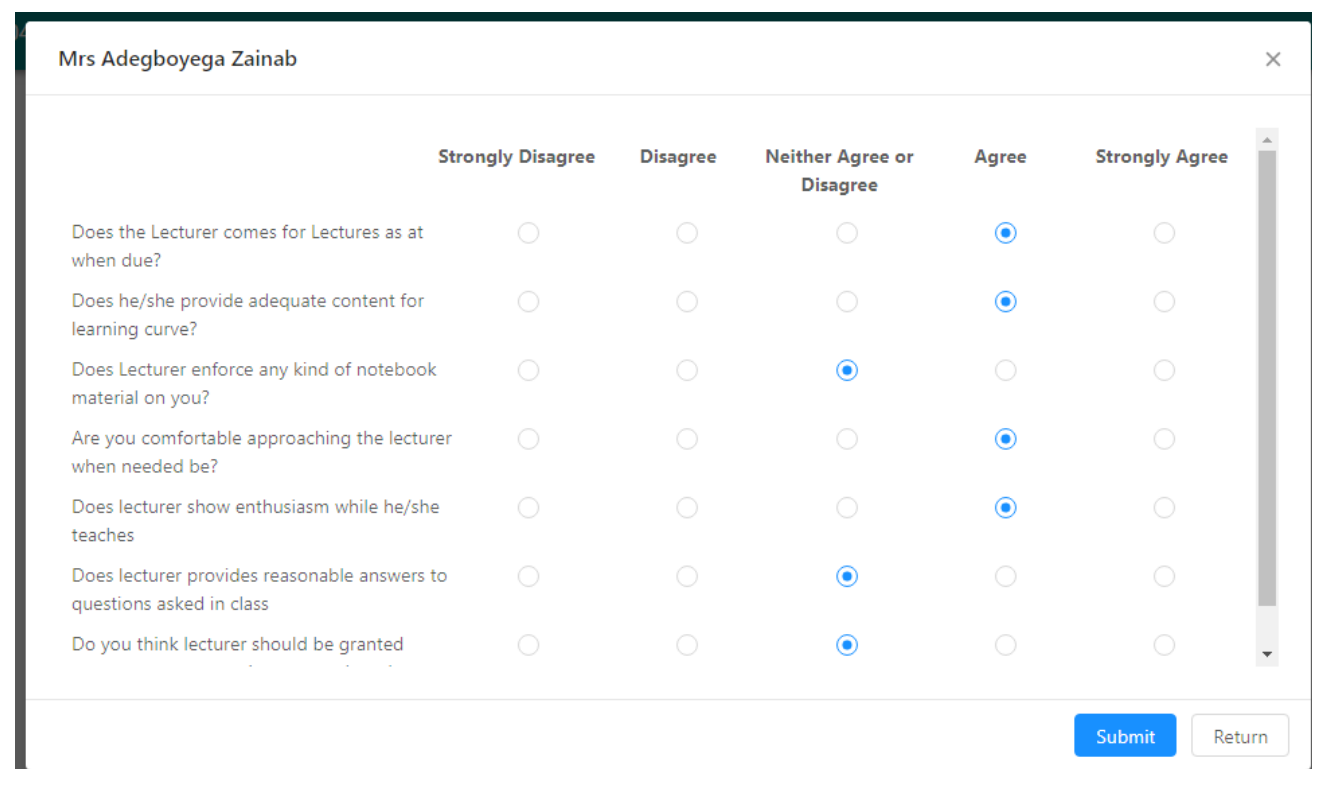

Figure 16: Question modal

\section{RESULT AND EVALUATION}

Over two hundred (200) academic staff across nine (9) departments in the faculty of a tertiary institution tested the proposed model between the month of October and November 2017. Evaluation of the model was carried out based on matching efficiency, attendance accuracy and the speed of capturing attendance and also performing authentication at the beginning and end of various class sessions. The matching efficiency parameters are presented as follows:

False Acceptance: Occurs when the wrong fingerprint is accepted as valid for an individual during verification. 
False Rejection: Occurs when the system fails to match the valid fingerprint of an individual.

True Acceptance: Occurs when a fingerprint matches with the fingerprint of same individual.

$$
\begin{array}{ll}
\text { FAR } & =\frac{F_{a}}{N} * 100 \\
\text { while FRR is computed as: } \quad F A R & =\frac{F_{r}}{N} * 100
\end{array}
$$

where $F_{a}=$ Number of False Acceptance, $F_{r}=$ Number of False Rejections and $\mathrm{N}=$ Number of verified objects (staff).
True Rejection: Occur when the system rejects a wrong fingerprint in the process of verifying an individual.

False Acceptance Rate (FAR) and False Reject Rate (FRR) are the error rates parameters used to express matching efficiency. FAR is computed as follows:

Table 1 shows the verification profile of some staff across the nine departments during class sessions.

\begin{tabular}{|c|c|c|c|c|c|c|c|c|}
\hline Staff ID & D.O.C & Dept & $\mathrm{F}_{\mathrm{a}}$ & $\mathrm{F}_{\mathrm{r}}$ & $\mathrm{T}_{\mathrm{a}}$ & $\mathrm{T}_{\mathrm{r}}$ & FAR\% & FRR\% \\
\hline adpersacse 922 & 3-10-2017 & $\mathrm{CSC}$ & 0 & 0 & 1 & 0 & 0.00 & 0.00 \\
\hline adpersacse 705 & 4-10-2017 & $\mathrm{CSC}$ & 0 & 0 & 1 & 0 & 0.00 & 0.00 \\
\hline adpersacse 801 & $5-10-2017$ & $\mathrm{CSC}$ & 0 & 0 & 1 & 0 & 0.00 & 0.00 \\
\hline adpersacse901 & 6-10-2017 & CSC & 0 & 0 & 1 & 0 & 0.00 & 0.00 \\
\hline adpersacse 787 & 9-10-2017 & $\mathrm{CSC}$ & 0 & 0 & 1 & 0 & 0.00 & 0.00 \\
\hline adpersacse 918 & 10-10-2017 & $\mathrm{CSC}$ & 0 & 0 & 1 & 0 & 0.00 & 0.00 \\
\hline adpersacse 811 & 11-10-2017 & $\mathrm{CSC}$ & 0 & 0 & 1 & 0 & 0.00 & 0.00 \\
\hline adpersacse 823 & 12-10-2017 & $\mathrm{CSC}$ & 0 & 0 & 1 & 0 & 0.00 & 0.00 \\
\hline adpersacse 912 & 13-10-2017 & $\mathrm{CSC}$ & 0 & 0 & 1 & 0 & 0.00 . & 0.00 \\
\hline adpersacse 862 & 16-10-2017 & $\mathrm{CSC}$ & 0 & 0 & 1 & 0 & 0.00 & 0.00 \\
\hline adpersacse 910 & $17-10-2017$ & $\mathrm{CSC}$ & 0 & 0 & 1 & 0 & 0.00 & 0.00 \\
\hline adpersacse 843 & 18-10-2017 & $\mathrm{CSC}$ & 0 & 0 & 1 & 1 & 0.00 & 0.00 \\
\hline adpersacse 763 & $23-10-2017$ & $\mathrm{BCH}$ & 0 & 0 & 1 & 0 & 0.00 & 0.00 \\
\hline adpersacse 885 & 24-10-2017 & $\mathrm{BCH}$ & 0 & 0 & 1 & 0 & 0.00 & 0.00 \\
\hline adpersacse 906 & $25-10-2017$ & $\mathrm{BCH}$ & 0 & 0 & 1 & 0 & 0.00 & 0.00 \\
\hline adpersacse 799 & $26-10-2017$ & PSB & 0 & 0 & 1 & 0 & 0.00 & 0.00 \\
\hline adpersacse 872 & $27-10-2017$ & PSB & 0 & 0 & 1 & 0 & 0.00 & 0.00 \\
\hline adpersacse 519 & $30-10-2017$ & $\mathrm{CHM}$ & 0 & 0 & 1 & 0 & 0.00 & 0.00 \\
\hline adpersacse699 & $31-10-2017$ & $\mathrm{CHM}$ & 0 & 0 & 1 & 0 & 0.00 & 0.00 \\
\hline adpersacse 617 & 01-11-2017 & $\mathrm{CHM}$ & 0 & 0 & 1 & 0 & 0.00 & 0.00 \\
\hline adpersacse 425 & 02-11-2017 & $\mathrm{CHM}$ & 0 & 0 & 1 & 0 & 0.00 & 0.00 \\
\hline adpersacse 480 & 03-11-2017 & $\mathrm{CHM}$ & 0 & 0 & 1 & 0 & 0.00 & 0.00 \\
\hline adpersacse 637 & 06-11-2017 & PHY & 0 & 0 & 1 & 0 & 0.00 & 0.00 \\
\hline adpersacse 955 & 06-11-2017 & PHY & 0 & 0 & 1 & 0 & 0.00 & 0.00 \\
\hline adpersacse 890 & 07-11-2017 & PHY & 0 & 0 & 1 & 0 & 0.00 & 0.00 \\
\hline adpersacse 441 & 08-11-2017 & PHY & 0 & 0 & 1 & 0 & 0.00 & 0.00 \\
\hline adpersacse 693 & 09-11-2017 & PHY & 0 & 0 & 1 & 0 & 0.00 & 0.00 \\
\hline adpersacse 560 & 10-11-2017 & AEB & 0 & 0 & 1 & 0 & 0.00 & 0.00 \\
\hline adpersacse 614 & 13-11-2017 & AEB & 0 & 0 & 1 & 0 & 0.00 & 0.00 \\
\hline adpersacse 709 & 14-11-2017 & AEB & 0 & 0 & 1 & 0 & 0.00 & 0.00 \\
\hline adpersacse939 & $15-11-2017$ & MATH & 0 & 0 & 1 & 0 & 0.00 & 0.00 \\
\hline adpersacse 500 & 16-11-2017 & MATH & 0 & 0 & 1 & 0 & 0.00 & 0.00 \\
\hline adpersacse 724 & 17-11-2017 & MATH & 0 & 0 & 1 & 1 & 0.00 & 0.00 \\
\hline
\end{tabular}

Table 1: Staff Verification Profile (SVP) 


\begin{tabular}{|c|c|c|c|c|c|c|c|c|}
\hline adpersacse918 & $20-11-2017$ & MATH & 0 & 0 & 1 & 0 & 0.00 & 0.00 \\
\hline adpersacse870 & $21-11-2017$ & MATH & 0 & 0 & 1 & 0 & 0.00 & 0.00 \\
\hline adpersacse794 & $22-11-2017$ & MATH & 0 & 0 & 1 & 0 & 0.00 & 0.00 \\
\hline adpersacse917 & $23-11-2017$ & GEY & 0 & 0 & 1 & 0 & 0.00 & 0.00 \\
\hline adpersacse809 & $24-11-2017$ & GEY & 0 & 0 & 1 & 0 & 0.00 & 0.00 \\
\hline adpersacse858 & $27-11-2017$ & GEY & 0 & 0 & 1 & 0 & 0.00 & 0.00 \\
\hline adpersacse701 & $28-11-2017$ & GPHY & 0 & 0 & 1 & 0 & 0.00 & 0.00 \\
\hline adpersacse533 & $28-11-2017$ & GPHY & 0 & 0 & 1 & 0 & 0.00 & 0.00 \\
\hline adpersacse464 & $29-11-2017$ & GPHY & 0 & 0 & 1 & 0 & 0.00 & 0.00 \\
\hline
\end{tabular}

Table Keys:

D.O.C $=$ Date of Capture

Dept $=$ Department

$\mathrm{F}_{\mathrm{a}}=$ False Acceptance

$\mathrm{F}_{\mathrm{r}}=$ False Rejections

$\mathrm{T}_{\mathrm{a}}=$ True Acceptance

$\mathrm{T}_{\mathrm{r}}=$ True Rejections

The results from Table 1 is presented as follows:
a. $\quad$ True Acceptance $=42$
b. True Rejection $=2$
c. False Acceptance Rate $(\mathrm{FAR})=0.00 \%$
d. False Rejection Rate $(F R R)=0.00 \%$

Evaluation of the proposed system's accuracy is presented in Table 2 as follows:

\begin{tabular}{|c|c|c|c|c|}
\hline Number of Staff & $\begin{array}{c}\text { Attendance not } \\
\text { counted }\end{array}$ & $\begin{array}{c}\text { Successful } \\
\text { Identification }\end{array}$ & $\begin{array}{c}\text { Unsuccessful } \\
\text { Identification }\end{array}$ & $\begin{array}{c}\text { Attendance } \\
\text { Accuracy }\end{array}$ \\
\hline 269 & 2 & 265 & 2 & $98.51 \%$ \\
\hline
\end{tabular}

The proposed system performed excellently with 98.51\% attendance accuracy based on the high successful staff identification recorded.

\section{CONCLUSION}

Discipline is one of the principles of management proposed by (Fayol, 1949) to carry out effective administration and management of an organization. It is a part of the core values of an organization that makes employees obey and respect the rules that governs the organization. Therefore, every academic institutions need to consider staff attendance irrespective of their responsibilities. This will ensure that academic staff deliver their lectures promptly with focus on quality content delivery. On the part of the University management, it will result to effective leadership. This research has provided adequate technology required to ensure that staff performance is promoted at a high rate. The system features a two-phase subsystem, an attendance system for staff enrollment and verification and a question-answering module to acquire students review on staff performance. Hence, the new system is effective, user friendly, reliable and provide high security which offers more to attendance management.

\section{REFERENCES}

[1] Adewole K. S., Abdulsalam S. O., Babatunde R. S., Shittu T. M., \& Oloyede M. O. (2014). Development of fingerprint biometric attendance system for non-academic staff in a tertiary institution. Computer Engineering and Intelligent Systems, 5(2), 62-70

[2] Eromosele, A.E. (2016). Student attendance monitoring system using fingerprint. Project Report submitted to the Department of Computer Science, Lagos State Polytechnic, Lagos.

[3] Siddiqui, F., Naik, M., Yadav, S., \& Sunesara, M. (2017). Wireless attendance system using fingerprint. Imperial Journal of Interdisciplinary Research, 3(5), 564566.

[4] Talaviya, G., Ramteke, R., \& Shete, A.K.. (2013). Wireless fingerprint based college attendance system using zigbee technology. International Journal of Engineering and Advanced Technology, 2(3), 201-203.

[5] Garcia, R.L., Alberola-Lopez C., Aghzoutb, O., \& RuizAlzola, J. (2003). Biometric identification systems. Signal Processing, 83, 2539-2557.

[6] Shakil, M.D. \& Nandi, R.N., (2013). Attendance management system for industrial worker using finger print scanner. Global Journal of Computer Science and 
Technology, Graphics \& Vision, 13(6). Available t: https://scholar.google.com/citations?user=SEdfaSYAAAAJ \&hl=en.

[7] Josphineleela, R. \& Ramakrishnan, M. (2012). An efficient automatic attendance system using fingerprint reconstruction technique. International Journal of Computer Science and Information Security, 10(3). Available at: https://arxiv.org/ftp/arxiv/papers/1208/1208.1672.pdf. [8] Benyo, Balazs, et al. (2012). Student attendance monitoring at the university using NFC. Wireless Telecommunications Symposium. Available at: https://www.researchgate.net/publication/261263604_Stude nt_attendance_monitoring_at_the_university_using_NFC.

[9] Afolalu, C.A., Tope-Oke, A., \& Atsadu, S. (2016). Towards an efficient biometric employee attendance system. International Journal of Research in Business Management, 4(2), 65-78.

[10] Obansola O. Y., Makinde O.E., Adeshina A.H., \& Adebayo O.B. (2016). Development of staff attendance management system using fingerprint biometric identification technique. Greener Journal of Social Sciences, 6(3), 055-069.

[11] Pankanti, S., Prabhakar, S., \& Jain, A.K. (2002). On the individuality of fingerprints. IEEE Transaction on Pattern Analysis and Machine Intelligence, 24(8), 10101025.
[12] Ezema, L.S., Joe-Uzuegbu, C.K.A., Eneh J.N, \& Amanze, I. (2015). Fingerprint Based Attendance Management System. International Journal of Scientific and Engineering Research, 6(7), 1623-1628.

[13] Dalah C.M. (2014). Secured employee attendance management system using fingerprint. IOSR Journal of Computer Engineering, 16(1), 32-37.

[14] Suryawanshi, V., Aundhakar, S., Mane, N., \& Kamble, R. (2017). Attendance system using fingerprint identification with GUI. International Journal of Engineering Development and Research, 5(2), 1-9.

[15] Kadam, D.B., Bhimasen, W., Shubham, S., \& Vicky, K. (2017). Attendance system using fingerprint identification with website designing and GUI. International Research Journal of Engineering and Technology, 04(03), 1879-1882.

[16] Olagunju, M., Adeniyi, A.E., \& Oladele, T.O. (2018). Staff attendance monitoring system using fingerprint biometrics. International Journal of Computer Applications, 179(21), 8-15.

[17] Ikuomola, A.J. (2015). Fingerprint-based authentication system for time and attendance management. British Journal of Mathematics \& Computer Science, 5(6), 735-747.

[18] Esther Rani.D \& Dr. J. John Raybin Jose. (2016). Fingerprint-based biometric authentication. International Journal of Computer Science and Mobile Computing, 5(9), 6-15. 